Neurosurg Focus 24 (5):E5, 2008

\title{
Evaluation of the role of Gamma Knife surgery in the treatment of craniopharyngiomas
}

\author{
Rupa Gopalan, B.S., Kasandra Dassoulas, B.S., Jessica Rainey, \\ Jonathan H. Sherman, M.D., And Jason P. Sheehan, M.D., Ph.D. \\ Department of Neurological Surgery, University of Virginia Health System, Charlottesville, Virginia
}

\begin{abstract}
$\checkmark$ The management of craniopharyngioma involves balancing adequate reduction in tumor volume and prevention of recurrence while minimizing damage to delicate surrounding structures. Because of the lesion's proximity to the optic chiasm and its relationship to the hypothalamic-pituitary axis, morbidity rates following treatment can be high. Gamma Knife surgery (GKS) is now being considered as a viable method of providing tumor control while ensuring minimal side effects. The authors conducted a literature review of 10 studies in which GKS was used to treat craniopharyngioma; some lesions had been previously treated and some had not. The mean marginal dose ranged from 5 to 16.4 Gy (mean 12.3 Gy). Tumor control was achieved in $75 \%$ of cases overall and varied with tumor subtype (cystic, solid, mixed). Control was seen in $90 \%$ of solid, $80 \%$ of cystic, and $59 \%$ of mixed tumors. The overall morbidity rate resulting from radiosurgery was $4 \%$ and the overall mortality rate was $0.5 \%$. These results suggest that GKS may provide a favorable benefit-to-risk profile for many patients with craniopharyngiomas.
\end{abstract}

(DOI: $10.3171 /$ FOC/2008/24/5/E5)

\section{KEY WORDS • craniopharyngiomas • Gamma Knife • radiosurgery}

$\mathrm{C}$ RANIOPHARYNGIOMAS are tumors of the parasellar region that arise from residual epithelial cells of the Rathke pouch. They may be solid, cystic, or mixed in nature and are commonly calcified. Up to $60 \%$ of craniopharyngiomas have both cystic and solid components. 7,28 Craniopharyngiomas occur in a bimodal age distribution, generally appearing in young patients between the ages of 5 and 14 years and in older patients between 50 and 74 years. According to an international meta-analysis of $>$ 10,000 individuals, craniopharyngiomas account for $\sim 7.8 \%$ of pediatric brain tumors. ${ }^{31}$ Because the tumor is relatively rare and generally benign, the results of demographic studies in adults are inconclusive.

Although histologically benign, the resulting clinical sequelae can be severe. Neurological symptoms can be divided into those resulting from mass effect and those resulting from dysfunction of the hypothalamic-pituitary axis. Mass effect can result in increased intracranial pressure presenting as headache, nausea, and vomiting. Obstruction of the aqueduct of Sylvius or the foramina of Monro may result in hydrocephalus in cases with large mass lesions. ${ }^{10}$ Hydrocephalus is a more common presenting sign in children than in adults. ${ }^{15}$ Due to its location in the sellar and parasellar areas, compression of the optic chiasm typically results in bitemporal hemianopsia. Visual disturbance can also include central visual defects, optic atrophy, and papilledema. Headache and visual field defects are the most common presenting symptoms. ${ }^{2,18,20}$ Endocrine disruption is

Abbreviations used in this paper: $\mathrm{CN}=$ cranial nerve; GKS = Gamma Knife surgery; GTR = gross-total resection. often suppressed, manifesting as amenorrhea, hypothyroidism, orthostatic hypotension, and diabetes insipidus, which occurs in as many as $15 \%$ of craniopharyngioma patients. ${ }^{9} 15$ In children, growth hormone deficiency may result in delayed puberty and short stature., ${ }^{1,22}$

Treatment options for craniopharyngioma include total or subtotal resection, external-beam radiation therapy, stereotactic radiation therapy, intracavitary radiation, or a combination of treatment modalities. More recently, GKS has been utilized as either a primary or secondary treatment for craniopharyngioma patients. Given the spectrum of treatments available for this tumor, proper management remains controversial. In this paper, we evaluate the current role of Gamma Knife radiosurgery in the neurosurgical treatment armamentarium for craniopharyngioma patients.

\section{Clinical Materials and Methods}

In this study we review the trends in treatment of craniopharyngiomas for both pediatric and adult populations. Ten major studies of outcomes after GKS for craniopharyngioma were evaluated. Case studies were not included. Studies were evaluated for age at presentation, prior resection, concurrent treatment with intracavitary irradiation or bleomycin, follow-up duration, marginal radiation, mortality and morbidity rates, tumor control, and tumor progression (Table 1).

\section{Results}

\section{Population Characteristics}

The patient populations each ranged from 5 to 98 cases 
R. Gopalan et al.

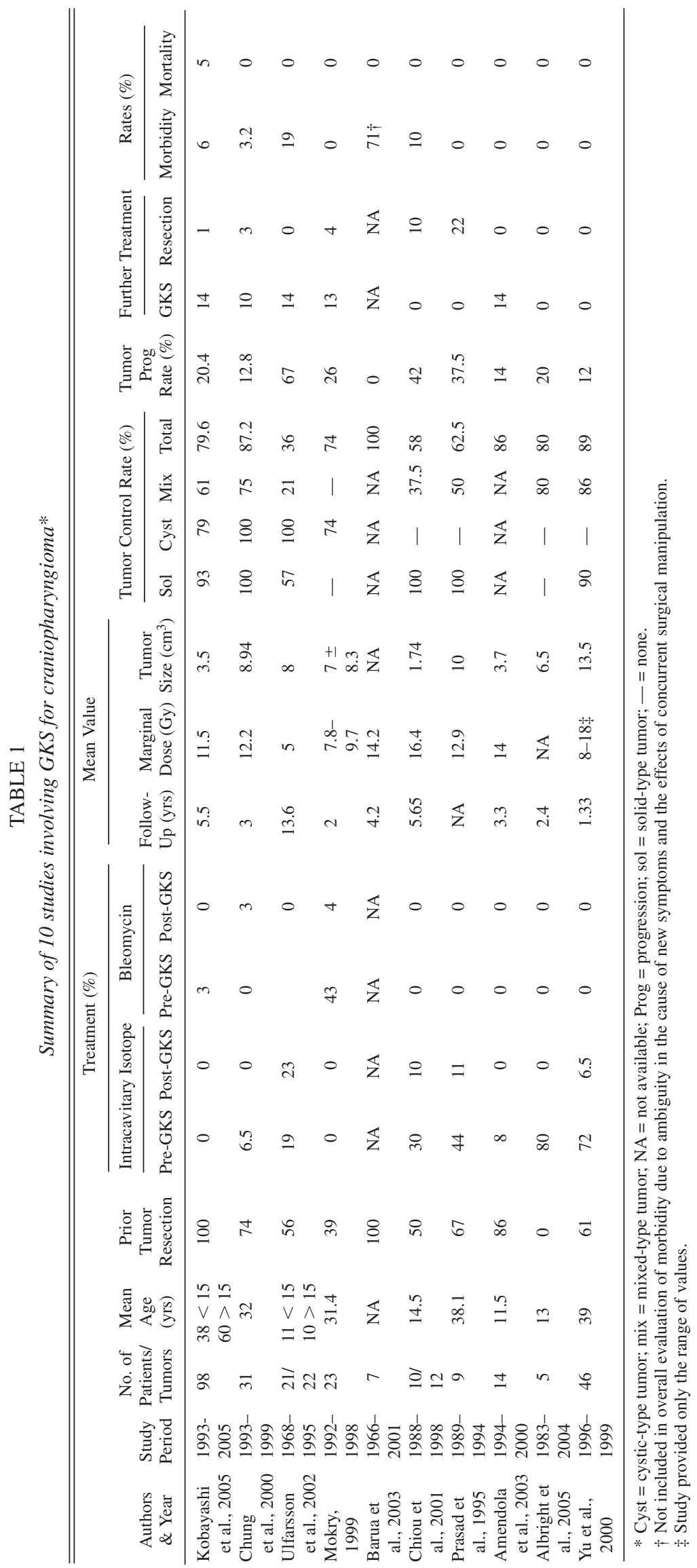


and included both children and adults. The average age of patients was 25.6 years (range 13-39 mean age). In 2 studies, tumor outcome was evaluated based on the number of tumors, as opposed to the number of patients. Kobayashi et al. ${ }^{19}$ and Ulfarsson et al. ${ }^{36}$ did not report mean age, but patients $>15$ years of age comprised 61 and $48 \%$ of these respective studies. Of the 10 studies reporting follow-up durations, the mean follow-up time was 4.6 years (range 1.33-13.6).

\section{Treatment Prior to GKS}

On average, in $63 \%$ (range $0-100 \%$ ) of cases, prior resection had been performed (either subtotal or complete). An average of $29 \%$ of patients underwent intracavitary radiotherapy prior to GKS. Patients in 2 studies were treated with bleomycin prior to GKS, $3 \%$ in the study by Kobayashi et al. ${ }^{19}$ and $43 \%$ in the study by Mokry. ${ }^{27}$

\section{Radiosurgical Dose}

On average, the mean marginal dose was $12.3 \mathrm{~Gy}$ (range 5-16.4 Gy). Mokry ${ }^{27}$ reported a mean marginal dose of 7.8-9.7 Gy, and Yu et al. ${ }^{38}$ reported a range of doses from 8-18 Gy (mean dose not available).

\section{Tumor Control and Progression}

All studies defined tumor control as a stable or decreased size of the tumor following GKS. Preoperative tumor sizes ranged from 1.74 to $13.5 \mathrm{~cm}^{3}$ (mean $7 \mathrm{~cm}^{3}$ ) and the mean size was not reported in 1 study. The mean tumor control rate was $75 \%$ (range 36-100\%). Solid tumors were treated in 6 studies and their mean control rate was 90\% (range 57-100\%); cystic tumors were treated in 4 studies and their mean control rate was $88 \%$ (range $74-100 \%$ ); and mixed tumors were treated in 7 studies and their mean control rate was $58.6 \%$ (range $21-86 \%$ ). Tumor progression was observed in $\sim 25 \%$ of cases (range $0-67 \%$ ).

\section{Further Treatment After GKS}

An average of $4 \%$ of patients underwent intracavitary radiotherapy following GKS. Chung et al. ${ }^{6}$ and Mokry ${ }^{27}$ treated patients with bleomycin following GKS to control cysts in 3 and $4 \%$ of patients, respectively. An average of $4 \%$ of patients (range $0-22 \%$ ) required an additional resection in an attempt to control tumor progression. An average of $7 \%$ of patients (range 0-14\%) underwent an additional treatment with GKS.

\section{Morbidity and Mortality Rates}

Assessment of morbidity included both endocrinological and visual deterioration resulting from GKS. Overall morbidity rates were low at 4\% (range 0-19\%). Death was only observed in 1 study and was reported as $5 \%$ of the population, giving an average mortality rate of $0.5 \%$ across all 10 studies. In $50 \%$ of the studies investigators reported no morbidity or mortality associated with GKS. Barua et al. ${ }^{3}$ reported a high incidence of morbidity, but this series was omitted from tabulation due to ambiguity in the underlying cause of the complications.

\section{Discussion}

\section{Resection for Craniopharyngiomas}

Traditional treatments for tumors of the sellar and parasellar regions include resection alone or resection coupled with radiosurgery or radiotherapy as an adjuvant treatment when complete resection is not possible. The proximity of craniopharyngiomas to the optic nerves, optic chiasm, oculomotor nerve, and carotid arteries, often precludes total resection. Although GTR is sometimes possible and should be the goal, it is often difficult and one must take into account the size, consistency (solid, cystic, or mixed), and location of the tumor in preoperative planning. Midline tumors with an origin below the diaphragm may be removed via a transsphenoidal route, either partially or completely. However, the transsphenoidal approach does not necessarily minimize postoperative endocrinopathy. 8,35

Morbidities associated with resection of craniopharyngiomas include visual deterioration and endocrine dysfunction. Visual deterioration is estimated to occur in an average of $19 \%$ of surgical cases, and it has been reported in as many as $35 \%$ of cases. ${ }^{4}$ Endocrine dysfunction results from damage to the hypothalamic-pituitary tract and is a major deficit following resection of craniopharyngiomas. Diabetes insipidus has been reported as the most common abnormality, seen in 59-93\% of cases. ${ }^{33}$ Honegger et al. ${ }^{13}$ have reported an increased incidence of diabetes insipidus from $16.1 \%$ preoperatively to $59.4 \%$ following resection. Many patients undergoing radical resection require longterm treatment for diabetes insipidus and additional hormone replacement therapy. ${ }^{4}$ Panhypopituitarism has been reported to occur in as many as $75-100 \%$ of patients undergoing resection. ${ }^{34}$

In a review of surgical series in which patients were treated for craniopharyngioma, Brada and Thomas ${ }^{4}$ reported a mean mortality rate of $12 \%$ (range $2-43 \%$ ) and a mean severe morbidity rate of $30 \%$ (range 12-61\%). A striking $40 \%$ (range $30-57 \%$ ) of the patients experienced hypothalamic morbidity. Authors at St. Jude's Children's Research Hospital found an increased incidence in both visual deterioration and endocrine dysfunction in the surgery-treated group compared with patients in whom more limited surgery and adjunctive radiotherapy were performed..$^{25}$ In a large-scale retrospective analysis of 309 patients treated by a single neurosurgeon, Shi et al. ${ }^{32}$ concluded that despite advances in imaging that have improved outcomes, morbidity related to hypopituitarism is still a major problem of craniopharyngioma surgery. This same study also reported a 3.9\% mortality rate associated with extirpation. Mark et al. ${ }^{24}$ reported a $10 \%$ mortality rate at 5 years and a severe surgical morbidity rate of $16 \%$. These authors further reported that all patients who had undergone complete resection had worsened functional status after surgery and that all patients in whom resection was attempted had worsened pituitary function.

In addition to the increased morbidity associated with tumor resection, tumor recurrence and the need for reoperation may arise despite an initial GTR. ${ }^{24}$ Prasad et al..$^{30}$ reviewed results of surgical treatment for craniopharyngioma published between 1966 and 1994 and reported that only $35.5 \%$ of these surgical series for craniopharyngioma achieved a radical resection of $>50 \%$ and that even when radical resection was accomplished, tumors recurred in 
$10-50 \%$ of cases. Although the utility of radiosurgery in treating craniopharyngiomas is still being analyzed in comparison to GTR, some studies have suggested that GTR is most strongly recommended in cases lacking hypothalamic involvement, whereas adjunctive or primary radiosurgery should be considered in other cases to decrease the rates of postoperative morbidity and mortality. ${ }^{1}$

Inoue et al. ${ }^{14}$ observed that GTR for craniopharyngioma causes more hypothalamic-pituitary dysfunction than radiosurgery and that radiosurgery-treated patients may have preserved function without the need for hormone replacement postoperatively. In a pediatric series of 66 patients, growth hormone deficiency was documented in $100 \%$ of cases, gonadotropin deficiency in $80 \%$, and hypothyroidism in $74 \%$ following either gross-total or partial craniopharyngioma resection. ${ }^{11}$ Radiotherapy following surgery did not increase the incidence of endocrine abnormalities, suggesting that the most of dysfunction following craniopharyngioma management results from surgery itself.

\section{Forms of Adjunctive Radiotherapy}

Several options exist for cases not amenable to GTR. Subtotal resection may be followed by conventional radiotherapy, intracavitary irradiation, fractionated radiotherapy, and stereotactic radiosurgery. Radiotherapeutic techniques have also been performed as a primary treatment modality in patients with no history of resection.

Fractionated Stereotactic Radiotherapy. Fractionated stereotactic radiotherapy delivers conventionally fractionated radiation with some degree of precision, and it has been suggested to provide increased tumor control with low toxicity. It involves the use of computed tomography and/or magnetic resonance imaging for treatment planning. The delivery of radiation is more localized than that achieved with conventional radiotherapy. A 5-year progression-free survival rate of $92 \%$ has been reported for fractionated stereotactic radiosurgery compared with $80-90 \%$ for complete excision and only $50-60 \%$ for partial resection. ${ }^{26}$ Despite its utility, fractionated stereotactic radiotherapy is still susceptible to several complications including vasculitis, neuropsychological changes, worsening of visual symptoms, and, rarely, an increased occurrence of secondary tumors. ${ }^{1}$

Stereotactic Intracavitary Irradiation. Stereotactic intracavitary radiation with yttrium-90 or phosphorus-32 betaemitting isotopes has been widely shown to be an effective primary treatment for multicystic or medium-sized monocytic tumors ${ }^{1,12,37}$ but not useful for solid tumors. ${ }^{12}$ This type of therapeutic approach has been shown to control up to $96 \%$ of cystic tumors ${ }^{23}$ and $88 \%$ when including all forms of partially cystic tumors. ${ }^{29}$ Side effects include blindness, $\mathrm{CN}$ dysfunction, new-onset diabetes insipidus, ${ }^{29}$ and panhypopituitarism. ${ }^{12,37}$ Vision may improve $(48 \%)$ in some patients but can deteriorate in others (29\%) following treatment. ${ }^{12}$ An additional limitation is the progression of solid elements or secondary cyst formation, requiring further treatment. ${ }^{23}$

\section{Primary or Secondary GKS as a Treatment for Craniopharyngioma}

Gamma Knife surgery involves stereotactic neuroimag- ing-based planning to deliver a single, precise fraction of radiation with a steep dose fall-off. The Leksell stereotactic frame offers a superior degree of patient immobilization compared with other types of radiotherapy.$^{17}$ The precision of the Gamma Knife typically allows for sparing of critical structures such as the hypothalamus and optic apparatus from damaging amounts of radiation. Several authors have reported that a therapeutic effect in craniopharyngiomas is seen with a marginal dose of $6 \mathrm{~Gy}$, but the optimal dose, while not definitive, probably lies between 9 and 12 Gy. ${ }^{6,19,36}$ Ulfarsson et al. ${ }^{36}$ noted that 2 patients with postoperative endocrine dysfunction had received marginal doses of $15 \mathrm{~Gy}$. The optic apparatus is usually restricted to a dose of $\leq 8 \mathrm{~Gy}{ }^{33}$ However, it has also been reported that up to 10 Gy may be tolerated. ${ }^{21}$ Other CNs within the cavernous sinus are thought to be more radioresistant than CN II.

Compared with other treatment modalities, GKS is associated with relatively few complications, avoiding the immediate complications of resection as well as some of the long-term problems associated with radiotherapy. ${ }^{34}$ In the 10 series we have reviewed, the mean morbidity rate was $4 \%$, which was considerably lower than the incidence of complications associated with resection and alternative forms of radiotherapy. Death was virtually absent and was only seen in a study by Kobayashi et al. ${ }^{27}$ in $5 \%$ of the cases. In a study of residual or recurrent craniopharyngiomas treated with GKS, Chiou et al. ${ }^{5}$ reported visual deterioration in a single patient $(10 \%)$.

Favorable quality of life outcome was most frequently demonstrated in patients with tumors that decreased in size following GKS.,19 Tumor regression after either initial or adjuvant GKS has been shown in some patients with craniopharyngiomas. In a study of 5 pediatric patients, GKS was the primary treatment for the solid portion of mixed tumors after ${ }^{32} \mathrm{P}$ intracavitary irradiation was used to treat the cystic portion. Two patients experienced tumor regression, and 2 experienced tumor stability. ${ }^{1}$ Studies in our review exhibited higher control rates in cases of single-type tumors, solid or cystic, than in mixed-type tumors. These results are consistent with the findings of Chung et al. ${ }^{6}$ who found that single-type tumors, either cystic or solid, responded more effectively than mixed-type tumors of a smaller volume.

Poor quality of life outcomes after GKS are associated with tumor progression. ${ }^{19}$ Mokry ${ }^{27}$ found that larger tumor volume and multicystic tumors were predictive of poor outcome. The best outcomes in his study were seen in patients with monocystic tumors treated with stereotactic drainage and bleomycin, followed by GKS. Ulfarsson et al. ${ }^{36}$ and Kobayashi et al. ${ }^{19}$ found that tumor progression after GKS tended to be a function of enlargement of the cystic rather than the solid component of the tumor. It has been suggested that tumors with large cysts treated with intracavitary irradiation may benefit from GKS after cyst size decreases. ${ }^{1}$ It is thought that solid-type tumors and the solid portions of mixed-type tumors are less responsive than cystic tumors to intracavitary irradiation. ${ }^{16}$ Prasad et al. ${ }^{30}$ have recommended that mixed-type tumors be treated with a combination of radioisotype instillation and GKS. The combined treatment of both GKS and intracavitary irradiation has been advocated as a primary therapy for mixed cystic-solid tumors. ${ }^{1,38}$

Possible complications of GKS include hypothalamic 
dysfunction resulting in changes in sleep and appetite, endocrine changes, visual defects, radiation necrosis, and malignant lesions. Complications resulting from radiation therapy vary depending on patient age, tumor size, and radiation doses. ${ }^{17}$ However, several authors have found that GKS provides excellent tumor control with minimal or no postoperative morbidity or mortality. ${ }^{1,25,6,6,19,27}$

Risk associated with GKS is elevated when a large cystic volume obscures the visibility of other structures, such as visual pathways, or if there is tumor adherence to radiosensitive structures. Numerous authors have suggested the use of multimodal treatments in such cases because large cystic or mixed-type tumors can be reduced through cyst aspiration. Such volume reduction may reduce the risk of radiation-induced injury and allow more effective targeting of the solid portion of the tumor. ${ }^{6,27}$ Proper dose planning and avoidance of optic pathways becomes possible. ${ }^{6}$ In addition, an immediate improvement in a patient's condition can occur following aspiration of a cystic-type tumor, as compression of critical neural structures is relieved.

\section{Conclusions}

Gamma Knife surgery provides reasonable benefit-torisk profile in the treatment of craniopharyngioma. In comparison to craniopharyngioma treated with resection alone, GKS appears to be associated with a decreased rate of morbidity and mortality. Due to great precision in tumor targeting, GKS may also be associated with fewer complications than other forms of radiation delivery. It can be used to treat residual or recurrent craniopharyngiomas after extirpation; it can also be used as an initial treatment option for some patients. Further studies will help to elucidate the role of GKS in the long-term management of craniopharyngioma in patients.

\section{References}

1. Albright AL, Hadjipanayis CG, Lunsford LD, Kondziolka D, Pollack IF, Adelson PD: Individualized treatment of pediatric craniopharyngiomas. Childs Nerv Syst 21:649-654, 2005

2. Amendola BE, Wolf A, Coy SR, Amendola MA: Role of radiosurgery in craniopharyngiomas: a preliminary report. Med Pediatr Oncol 41:123-127, 2003

3. Barua KK, Ehara K, Kohmura E, Tamaki N: Treatment of recurrent craniopharyngiomas. Kobe J Med Sci 49:123-132, 2003

4. Brada M, Thomas DG: Craniopharyngioma revisited. Int J Radiat Oncol Biol Phys 27:471-475, 1993

5. Chiou SM, Lunsford LD, Niranjan A, Kondziolka D, Flickinger JC: Stereotactic radiosurgery of residual or recurrent craniopharyngioma, after surgery, with or without radiation therapy. Neuro Oncol 3:159-166, 2001

6. Chung WY, Pan DH, Shiau CY, Guo WY, Wang LW: Gamma knife radiosurgery for craniopharyngiomas. J Neurosurg 93 (3 Suppl):47-56, 2000

7. Coffey R, Lunsford LD: The role of stereotactic techniques in the management of craniopharyngiomas. Neurosurg Clin N Am 1: $161-172,1990$

8. Frank G, Pasquini E, Doglietto F, Mazzatenta D, Sciarretta V, Farneti G, et al: The endoscopic extended transsphenoidal approach for craniopharyngiomas. Neurosurgery 59 (1 Suppl): ONS75-ONS83, 2006

9. Garnett MR, Puget S, Grill J, Sainte-Rose C: Craniopharyngioma. Orphanet J Rare Dis 2:18, 2007

10. Garrè ML, Cama A: Craniopharyngioma: modern concepts in pathogenesis and treatment. Curr Opin Pediatr 19:471-479, 2007

11. Gonc EN, Yordam N, Ozon A, Alikasifoglu A, Kandemir N: Endocrinological outcome of different treatment options in children with craniopharyngioma: a retrospective analysis of 66 cases. Pediatr Neurosurg 40:112-119, 2004

12. Hasegawa T, Kondziolka D, Hadjipanayis CG, Lunsford LD: Management of cystic craniopharyngiomas with phosphorus-32 intracavitary irradiation. Neurosurgery 54:813-822, 2004

13. Honegger J, Buchfelder M, Fahlbusch R: Surgical treatment of craniopharyngiomas: endocrinological results. J Neurosurg 90: 251-257, 1999

14. Inoue HK, Fujimaki $\mathrm{H}$, Kohga $\mathrm{H}$, Ono $\mathrm{N}$, Hirato $\mathrm{M}$, Ohye $\mathrm{C}$ : Basal interhemispheric supra- and/or infrachiasmal approaches via superomedial orbitotomy for hypothalamic lesions: preservation of hypothalamo-pituitary functions in combination treatment with radiosurgery. Childs Nerv Syst 13:250-256, 1997

15. Jane JA Jr, Laws ER: Crainopharyngioma. Pituitary 9:323-326, 2006

16. Julow J, Backlund EO, Lányi F, Hajda M, Bálint K, Nyáry I, et al: Long-term results and late complications after intracavitary yttrium-90 colloid irradiation of recurrent cystic craniopharyngiomas. Neurosurgery 61:288-296, 2007

17. Kalapurakal J: Radiation therapy in the management of pediatric craniopharyngiomas—a review. Childs Nerv Syst 21:808-816, 2005

18. Karavitaki N, Brufani C, Warner JT, Adams CB, Richards P, Ansorge O, et al: Craniopharyngiomas in children and adults: systematic analysis of 121 cases with long-term follow-up. Clin Endocrinol (Oxf) 62:397-409, 2005

19. Kobayashi T, Kida Y, Mori Y, Hasegawa T: Long-term results of gamma knife surgery for the treatment of craniopharyngioma in 98 consecutive cases. J Neurosurg 103 (6 Suppl):482-488, 2005

20. Larijani B, Bastanhagh MH, Pajouhi M, Kargar Shadab F, Vasigh A, Aghakhani S: Presentation and outcome of 93 cases of craniopharyngioma. Eur J Cancer Care (Engl) 13:11-15, 2004

21. Leber KA, Berglöff J, Pendl G: Dose-response tolerance of the visual pathways and cranial nerves of the cavernous sinus to stereotactic radiosurgery. J Neurosurg 88:43-50, 1998

22. Levy A: Pituitary disease: presentation, diagnosis, and management. J Neurol Neurosurg Psychiatry 75 (3 Suppl): iii47-iii52, 2004

23. Lunsford LD, Pollock BE, Kondziolka DS, Levine G, Flickinger JC: Stereotactic options in the management of craniopharyngioma. Pediatr Neurosurg 21 (1 Suppl):90-97, 1994

24. Mark RJ, Lutge WR, Shimizu KT, Tran LM, Selch MT, Parker, RG: Craniopharyngioma: treatment in the CT and MR imaging era. Radiology 197:195-198, 1995

25. Merchant TE, Kiehna EN, Sanford RA, Mulhern RK, Thompson SJ, Wilson MW, et al: Craniopharyngioma: the St. Jude Children's Research Hospital experience 1984-2001. Int J Radiat Oncol Biol Phys 53:533-542, 2002

26. Minniti G, Saran F, Raish D, Soomal R, Sardell S, Gonsalves A, et al: Fractionated stereotactic conformal radiotherapy following conservative surgery in the control of craniopharyngiomas. Radiother Oncol 82:90-95, 2007

27. Mokry M: Craniopharyngiomas: a six year experience with Gamma Knife radiosurgery. Stereotact Funct Neurosurg 72 (1 Suppl):140-149, 1999

28. Musolino A, Munari C, Blond S, Betti O, Lajat Y, Schaub C, et al: [Stereotactic treatment for expanding cysts in craniopharyngiomas by endocavitary beta-irradiation (186-re; 198-au; 90-y).] Neurochirurgie 31:169-178, 1985 (Fr)

29. Pollock BE, Lunsford LD, Kondziolka D, Levine G, Flickinger JC: Phosphorus-32 intracavitary irradiation of cystic craniopharyngiomas: current technique and long-term results. Int J Radiat Oncol Biol Phys 33:437-446, 1995

30. Prasad D, Steiner M, Steiner L: Gamma knife surgery for craniopharyngioma. Acta Neurochir (Wien) 134:167-176, 1995 
31. Rickert $\mathrm{CH}$, Paulus W: Epidemiology of central nervous system tumors in childhood and adolescence based on the new WHO classification. Childs Nerv Syst 17:503-511, 2001

32. Shi XE, Wu B, Fan T, Zhou ZQ, Zhang YL: Craniopharyngioma: surgical experience of 309 cases in China. Clin Neurol Neurosurg 110:151-159, 2008

33. Stephanian E, Lunsford LD, Coffey RJ, Bissonette DJ, Flickinger JC: Gamma knife surgery for sellar and suprasellar tumors. Neurosurg Clin North Am 3:207-218, 1992

34. Suh JH, Gupta N: Role of radiation therapy and radiosurgery in the management of craniopharyngiomas. Neurosurg Clin N Am 17: 143-148, 2006

35. Teo C: Application of endoscopy to the surgical management of craniopharyngiomas. Childs Nerv Syst 21:696-700, 2005

36. Ulfarsson E, Lindquist C, Roberts M, Rähn T, Lindquist M, Thorén $\mathrm{M}$, et al: Gamma knife radiosurgery for craniopharyn- giomas: long-term results in the first Swedish patients. J Neurosurg 97 (5 Suppl):613-622, 2002

37. Voges J, Sturm V, Lehrke R, Treuer H, Gauss C, Berthold F: Cystic craniopharyngioma: long-term results after intracavitary irradiation with stereotactically applied colloidal beta-emitting radioactive sources. Neurosurgery 40:263-270, 1997

38. Yu X, Liu Z, Li S: Combined treatment with stereotactic intracavitary irradiation and gamma knife surgery for craniopharyngiomas. Stereotact Funct Neurosurg 75:117-122, 2000

Manuscript submitted December 18, 2007.

Accepted February 14, 2008.

Address correspondence to: Jason Sheehan, M.D., Ph.D., Department of Neurological Surgery, Box 800-212 University of Virginia Health System, Charlottesville, Virginia 22908. email: jps2f @ virginia.edu. 